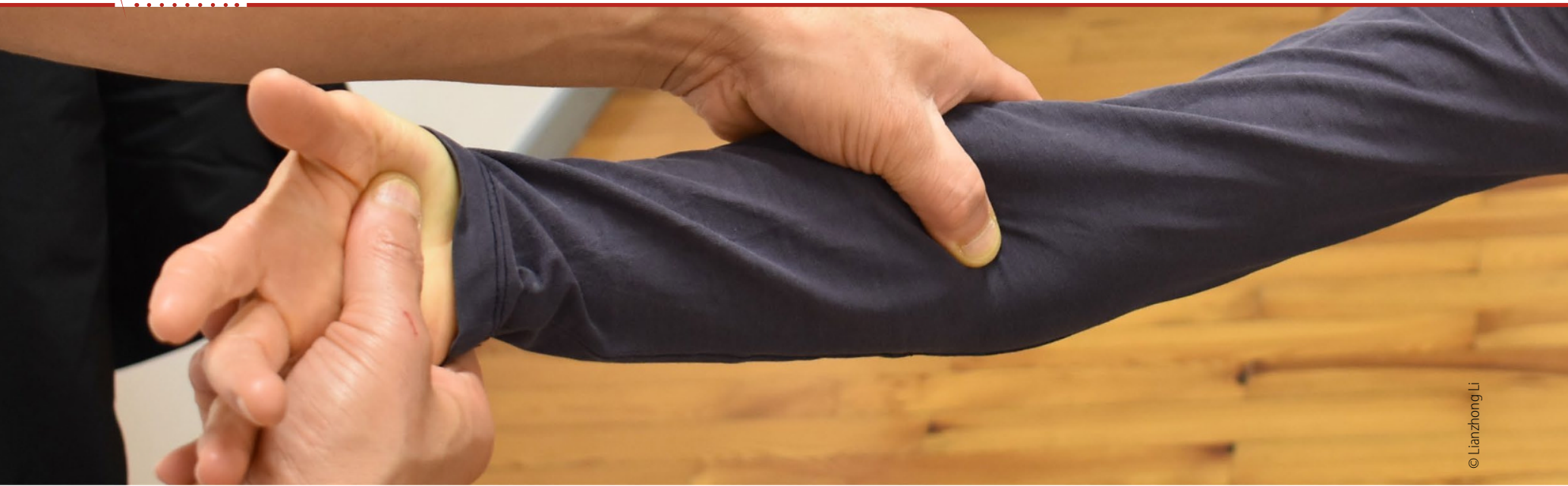

\title{
Die interprofessionelle Integration chinesischer Handgrifftechniken
}

\author{
Tuina im Spannungsfeld der Professionen
}

\section{Tuina - was ist das Besondere?}

\section{Säule der TCM}

Die chinesische Manualtherapie ist eine der Säulen im therapeutischen System der chinesischen Medizin. Die Hände des Arztes oder Therapeuten werden dazu benutzt, um mit Berührung und verschiedentlich auch Bewegungen Energie zu übertragen und eine mechanische Einwirkung zu erzeugen. Die gebräuchlichsten chinesischen Bezeichnungen für die chinesische Manualtherapie sind Tuina und Anmo. Sie setzen sich aus Bezeichnungen für einzelne Grifftechniken zusammen, dabei steht die Grundbedeutung von „tui“ für Schieben, „na“ bezeichnet Greifen, Ziehen und Nehmen, „an“ Drücken und „mo“ Reiben.

\section{Äußere Behandlung}

Die chinesische Manualtherapie und die Akupunktur haben gemein, dass das me-

\section{Deutsche Zeitschrift für Akupunktur}

$2020 \cdot 63$ (1): 12-15

https://doi.org/10.1007/s42212-020-00248-6

(c) Springer Medizin Verlag $\mathrm{GmbH}$, ein Teil von

Springer Nature 2020 thodische Prinzip der Therapie die Beeinflussung innerer Vorgänge des Körpers über äußere Strukturen, die Haut und auch myofasziale Weichteile darstellt. Auch die wesentlichen Theoreme der chinesischen Medizin, beispielsweise die Lehre von den Leitbahnen, die Lehre von Qi und Blut oder die Lehre der Zangfu (innere Organe) sind gemeinsamer Bestand ihres theoretischen Gebäudes. Der Zugriff auch auf besondere Punkte im Körper des Menschen, die uns als Akupunkturpunkte geläufig sind, ist spezifisch für die chinesische Tradition manueller Therapie, die im Übrigen in vielen verschiedenen Kulturen nachweisbar ist.

\section{Weichteiltechniken und Manipulationen}

Vor 25 Jahren hatte ich als Facharzt und in Ausbildung befindlicher Lehrer der Chirotherapie das Glück und die Ehre, zusammen mit H. D. Wolff, einem Altmeister der Chirotherapie, einen Kursus der mitteleuropäischen manuellen Medizin in Beijing auszurichten (Abb. 1 und Abb. 2). Gerade im Rahmen meiner Aufgabe der Übersetzung und Rückübersetzung wurde deutlich, dass in der heutigen Anwendung der Manualtherapie in
China die Vorstellungen und Begriffe der Biomechanik prägend sind. Dies gilt sowohl für die Manipulation am Achsenorgan, die man sich als Reposition einer Subluxation vorstellt, als auch für Publikationen zur Anwendung von Tuina beispielsweise in der Therapie von lumbalen Bandscheibenverlagerungen mit dem Ziel, diese rückgängig zu machen [1]. Mögen auch die Vorstellungen von den zugrunde liegenden therapeutischen Prinzipien differieren, die Tuina hat eine Reihe von Handgrifftechniken in ihrem Repertoire, die nicht nur ein originäres Alleinstellungsmerkmal beanspruchen können, sondern auch in hoher Versatilität die Brücke zu einer kombinierten Anwendung mit der Akupunktur schlagen können.

\section{Breites Anwendungsspektrum}

Die chinesische Manualtherapie umfasst dabei wie jede manuelle Therapie auch ein breites Spektrum der Anwendung, wie sich anhand einer Liste der verschiedenen Formen der Massage Anmo ermessen lässt: Von der Gesunderhaltungsmassage („bao jian an mo“) über die Leitbahnmassage (,jing luo an mo") und traumatologische Handgriffe der Frakturbehandlung 


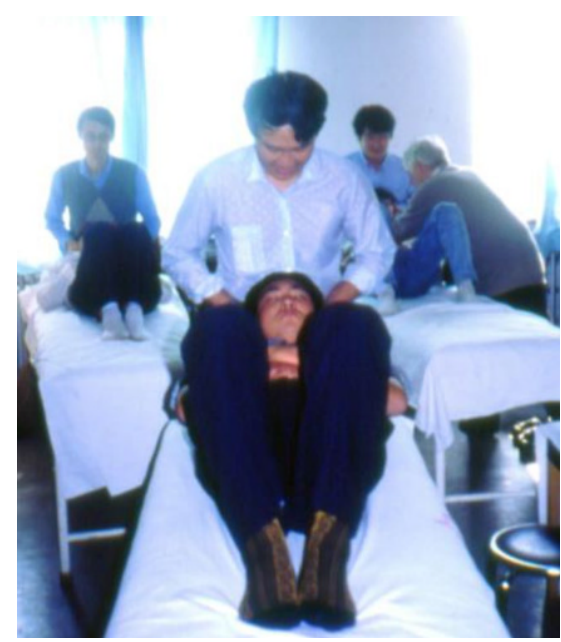

Abb. 1 Kurs Manuelle Medizin für chinesische Tuina-Therapeuten, Beijing Oktober 2004. (Mit freundl. Genehmigung, () J. Bachmann, alle Rechte vorbehalten)

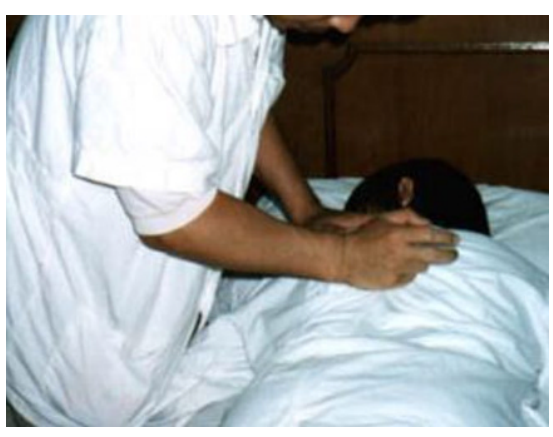

Abb. 2 Gun-Fa, Rollen, eine anspruchsvolle originäre Handgrifftechnik, versatil und gut kombinierbar mit Akupunktur. (Mit freundl. Genehmigung, ( ) J. Bachmann, alle Rechte vorbehalten)

(„zhenggu“), die Kinder-Tuina (siehe die Buchrezension in diesem Heft) bis zur Entspannungsmassage („fang song an mo“) spannt sich ein weiter Bogen, auch wenn Indikationen am Bewegungssystem mit Funktionsstörungen und Schmerzen im Vordergrund stehen.

\section{Fertigkeitsorientierung}

Es handelt sich somit um eine Tradition, in der eine Orientierung auf Fertigkeiten gefragt ist, die nicht ohne persönliche Unterweisung und Übung auskommt, d. h., neben theoretischen Voraussetzungen sind Familientraditionen und die persönlichen, auch körperlichen Eigenschaften des Therapeuten wesentlich für die konkrete Ausübung. Da die insoweit verschiedenen Schulen der manuellen Therapie aber alle bestrebt sind, ohne Verletzung der körperlichen Integrität und in einfacher Weise die körpereigenen Regulationsmechanismen und Reflexe zu nutzen, spielen natürlich auf der anderen Seite auch die Konstitution und Kondition des Patienten eine entscheidende Rolle für die konkrete und korrekte Anwendung.

\section{Stand der Erkenntnisse}

\section{Standards und Standardisierung}

Vor dem Hintergrund der verstärkten Inanspruchnahme komplementärer und alternativer Medizin (CAM) hat die Weltgesundheitsorganisation (WHO) in einer Sitzung im November 2007 Maßstäbe für die Ausbildung als Tuina-Therapeut formuliert und 2010 veröffentlicht [2]. Beschlüsse einer Folgesitzung im August 2019 harren noch der Veröffentlichung [3].

Gleichzeitig ist es seit Langem das Bestreben, die Handgrifftechniken standardisiert zu unterweisen - dies habe ich selbst in Shanghai 1989 so erfahren - und mit verschiedenen technischen Hilfsmitteln einer Messbarkeit und Prüfung zuzuführen [4].

Vor dem Hintergrund der Eingangsbemerkungen zur Begriffsbestimmung besteht aber noch kein Anlass, die Tuina-Therapie als standardisierte oder gar als eine Intervention anzusehen, die sich für eine Verblindung im Rahmen einer randomisierten klinischen Studie eignen würde. Dies erschwert die Methodik der klinischen Forschung und schränkt die Belastbarkeit ihrer Ergebnisse ein.

\section{Studienlage und Stand der Forschung}

Die Tuina-Therapie ist in ihrem Heimatland in der Versorgung etabliert, dementsprechend gibt es klinische Studien zu der Frage, inwieweit im Rahmen der Behandlung von chronischen unspezifischen Rückenschmerzen die zusätzliche Anwendung von Übungen zur Tiefenstabilisation einen therapeutischen Nutzen ergibt [5]. Für das Erkenntnisinteresse aus Sicht der hiesigen physikalischen Therapie und Rehabilitation wäre dieser Ansatz auf den Kopf zu stellen: Welche Auswirkungen hat die zusätzliche Anwendung von Tuina im Kontext aktivierender Ansätze und stabilisierender Übungen? Gleichwohl wird auch von chinesischer Seite das breite methodische Spektrum in der Rückenschmerzbehandlung narrativ dargestellt [6]. Einen erweiterten Blick auf die Studienlage und das so gekennzeichnete Potenzial wirft der Beitrag von Fleckenstein.

\section{Prinzipien der klinischen Anwendung und Fallbeispiel}

Die Prinzipien der klinischen Anwendung sind im Heft 2 aus 2019 am Beispiel der Schulter vor dem Hintergrund einer Ausbildung in den 1980er-Jahren in Beijing und Shanghai dargestellt worden. Im vorliegenden Heft widmet sich der Beitrag von Li Lian Zhong der Behandlung im Bereich der Halswirbelsäule, dies vor dem Hintergrund einer langjährigen Erfahrung in allen Facetten der Triggertherapie und auf dem Boden einer fundierten Ausbildung in Jinan, Shandong. Anhand der Fallbeispiele zur Fazialisparese in diesem Heft werden die verschiedenen Behandlungsansätze der Akupunktur und ihrer Kombination mit Tuina bei akuten (Uy) und eher chronischen Verläufen (Li) exemplarisch entwickelt und einer vergleichenden Würdigung zugänglich. Gleichzeitig lässt sich ableiten, dass die Tuina-Therapie nicht auf die Anwendung am Bewegungssystem beschränkt ist, sondern auch starke neurologische Indikationen hat.

\section{Recht und Geld}

\section{Interprofessionelle Arbeitsteilung}

Im chinesischen Gesundheitssystem wurde Tuina in der Versorgung augenscheinlich sowohl von Ärzten der chinesischen Medizin, gegebenenfalls mit zusätzlicher Fachausbildung, wie zum Beispiel „Orthopädie und Traumatologie" oder auch „Tuina und Akupunktur", erbracht, aber auch verordnet und diese Verordnung durch Tuina-Therapeuten umgesetzt. Diese Arbeitsteilung ist für den Kontext der deutschen und europäischen medizinischen Versorgung noch nicht definiert. Schließlich geht es neben der ökonomischen Darstellbarkeit auch um die Verteilung von Definitionsmacht, d. h., wer stellt die Diagnose, wer trägt die Verantwortung und hat daher die Haftpflicht. Schließlich bedarf es einer Abgrenzung zu nichttherapeutischen Anwendungen im Rahmen der Wellness. Der Beitrag von Roland Warther zeigt solche rechtlichen Aspekte auf - auch da, wo man sie gar nicht vermutet hätte, wie z. B. der Bauordnung. 


\section{Diagnose und Indikation}

In der Versorgung durch die gesetzliche Krankenversicherung in Deutschland ist die Rollenverteilung zwischen Arzt und Therapeuten geregelt: Manuelle Therapie ist als Rubrik der Krankengymnastik ein verordnungsfähiges Heilmittel. Für die Verordnung bedarf es keiner zusätzlichen ärztlichen Qualifikation. Bislang besteht für die chinesische Manualtherapie weder eine Verordnungsfähigkeit noch eine definierte ärztliche Qualifikation zur Verordnung oder Ausübung. Nach hiesiger Auffassung handelt es sich aber sicherlich um eine Form der Therapie mit Indikationen und Kontraindikationen. Der Versuch, die Tuina-Therapie analog zur thailändischen Massage in den Bereich der Wellness zu definieren, erscheint höchst problematisch. Wesentliche Inhalte, Erkenntnisse und therapeutische Optionen würden unterschlagen oder fehlgedeutet. Haftungsrechtliche Fragen würden sich in gänzlich anderem Rahmen bewegen.

\section{Risiken und Nebenwirkungen}

Manuelle Medizin bezeichnet eine ärztliche Zusatzweiterbildung, die Mitte der 1970er-Jahre unter der Bezeichnung Chirotherapie eingeführt wurde. Der Einführung der Zusatzbezeichnung ging ein Prozess der Überprüfung chiropraktischer Techniken voran. Dabei sind gerade Techniken mit hoher Geschwindigkeit und Manipulationen unter Aspekten der Patientensicherheit ausgiebig diskutiert und einem Ausleseprozess unterworfen worden. Sie stehen gleichzeitig für die differenzierte Anwendung durch Ärzte bzw. Physiotherapeuten, wobei die Fokussierung auf die Manipulation einer der Faktoren gewesen sein mag, dass sich im Rahmen einer Gegenbewegung ein stärkeres Interesse an weichen und sanften Techniken und auch einem umfassenderen Krankheitsverständnis entwickelte, wie es die Osteopathie anbietet. Dies verhält sich analog zu den Möglichkeiten, die in der chinesischen Manualtherapie stecken. Ihre Stärken liegen in den differenzierten Weichteiltechniken und in der konzeptionellen Berücksichtigung von Fernwirkungen gemäß dem Modell der Leitbahnen in der Akupunktur. Die Attraktivität der Tuina ist allerdings mit der Einschränkung behaftet, dass die Manipulationstechniken keinen gleichermaßen gestalteten Prozess der Überprüfung durchlaufen haben und sich die traditio- nelle Diagnose- und Indikationsstellung vornehmlich auf die sinnliche Wahrnehmung und Interaktion mit dem Patienten stützte - bei aller Wertschätzung dieser Verfahrensweise beinhaltet sie dennoch Wahrnehmungslücken, die beispielsweise durch bildgebende Verfahren geschlossen werden könnten.

\section{Therapeuten, Zeitbindung und Gebührenordnung}

Die therapeutische Sitzung der chinesischen Manualtherapie erfordert in der Anwendung lokaler, regionaler Techniken sowie beim Einsatz von umfassenden Stimulationen der Akupunkturpunkte an den Extremitäten ununterbrochen therapeutische Präsenz. Dies unterscheidet sie von der Akupunktur. Der zeitliche Aufwand hat Eingang in die Vorschläge der DÄGfA zur Novellierung der GOÄ gefunden. Dennoch bleibt abzuwarten, inwieweit die kommende Gebührenordnung einen Rahmen schafft, in dem sich die ärztliche Behandlung mit chinesischer Manualtherapie darstellen lässt.

\section{Tuina - ist die chinesische} Manualtherapie integrierbar?

Empirisch gelingt es dem Autor regelmäßig, im Einzelfall Handgrifftechniken der Tuina und die Akupunktur kombiniert und mit Erfolg einzusetzen. Hierfür eignet sich beispielsweise das Rollen, Gun 滚. Auch hilft die Schulung manueller Fertigkeiten in der Palpation von Akupunkturpunkten und der Durchführung von komplexeren Nadelstimulationstechniken.

Eine systematische Ausarbeitung oder gar Integration der Tuina als eigenständiger Säule der chinesischen Medizin steckt aber noch in den Kinderschuhen.

\section{》) Mit der Osteopathie liegt ein analoges Beispiel zur Tuina vor}

Für prognostische Einschätzungen $\mathrm{zu}$ solchen neuen Entwicklungen erscheint es immer wieder hilfreich, nach ähnlich gelagerten Problemstellungen zu suchen und deren Entwicklung und Dynamik aus den Blickwinkeln der verschiedenen Akteure zu betrachten. Für die chinesische Manualtherapie, Tuina, liegt ein solches, in mehrfacher Hinsicht analoges Beispiel mit der in den letzten Jahren zunehmend verbreiteten Osteopathie vor. Auch hier geht es um einen Kulturtransfer, in diesem Fall aus einem Kontext eigenständiger, universitärer Entwicklung in den USA, in die Verhältnisse einer regulierten medizinischen Versorgung hier. Die vielfältigen Aspekte einer Integration volksmedizinischer, alternativmedizinischer und komplementärmedizinischer Verfahren tangieren die verschiedenen professionellen heilberuflichen Akteure ganz unterschiedlich: Die Sicht des Manualtherapeuten und die Sicht des Manualmediziners oder Orthopäden und die Sicht des Osteopathen sind sicher nicht einheitlich. Auch stellen sich auf der anderen Seite der therapeutischen Beziehung Fragen der Patientensicherheit, der Patientenbedürfnisse und -interessen, die noch einer weiteren Bearbeitung bedürfen.

\section{Zusammenfassung}

Nach persönlicher Erfahrung fördern manuelle Fertigkeiten die Akupunkturtechnik und die Integration manueller Therapieformen das Behandlungsergebnis und finden im Einzelfall Anwendung, so auch in den Fallbeispielen in diesem Schwerpunkt. Unter therapeutischen, medizinisch-wissenschaftlichen, aber auch unter juristischen und ökonomischen Aspekten bestehen für eine systematische Einführung und Integration der chinesischen Manualtherapie, Tuina, in das Behandlungsspektrum der hiesigen Versorgung noch eine ganze Reihe offener Fragen. Die wesentlichen Komplexe dieser Fragen werden einleitend zu den weiteren, vertiefenden Beiträgen in diesem Schwerpunkt erörtert.
Literatur

1. Yajun L (2002) Treatment of protrusion of the lumbar intervertebral disc by TCM massage. J Tradit Chin Med 22(2):126-127

2. World Health Organization (2010) Benchmarks for training in traditional/complementary and alternative medicine: benchmarks for training in tuina

3. https://Www.icmart.org/home/home.html, abgerufen 28. Dez. 2019

4. LeiF (2013) Research progress on the standardization of Chinese tuina therapy: a short review. Chin J IntegrMed 19(1):68-72 
5. Zhang Y, Tang S, Chen G, Liu Y (2015) Chinese massage combined with core stability exercises for nonspecific low back pain: a randomized controlled trial. Complement Ther Med 23(1):1-6. https://doi.org/10.1016/j.ctim.2014.12.005

6. Zhang B, Xu H, Wang J, Liu B, Sun G (2017) A narrative review of non-operative treatment, especially traditional Chinese medicine therapy, for lumbar intervertebral disc herniation. BioSci Trends 11(4):406-417. https://doi.org/10.5582/ bst.2017.01199

\section{Korrespondenzadresse}

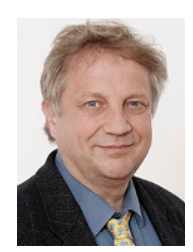

Dr. Jürgen Bachmann

Schmerzmedizin - Ortho-

pädie - Translationale Medizin

Augustastraße $25 \mathrm{c}$,

45525 Hattingen

Deutschland

dza@dr-bachmann-hattingen. de

Interessenkonflikt. J. Bachmann gibt an, dass kein Interessenkonflikt besteht.

Hier steht eine Anzeige.

刑 Springer 